\title{
Rag2 Deficiency Enhances Susceptibility to Systemic Mouse Adenovirus Type 1 Infection
}

\author{
Han-Kyul Lee ${ }^{a, b}$ Sun-Min Seo ${ }^{a}$ Jun-Young Kim ${ }^{a, c}$ Han-Woong Kim ${ }^{\text {a, } d}$ \\ Eui-Suk Jeong ${ }^{e}$ Yang-Kyu Choi ${ }^{a}$ \\ aDepartment of Laboratory Animal Medicine, College of Veterinary Medicine, Konkuk University, Seoul, Republic \\ of Korea; 'b Pathology Team, Biotoxtech Co. Ltd, Cheongju, Republic of Korea; 'Green Cross Corporation, Yongin-si, \\ Republic of Korea; ${ }^{d}$ Regenerative Dental Medicine Institute, Hysensbio, Gwacheon-si, Republic of Korea; 'Laboratory \\ Animal Center, Daegu-Gyeongbuk Medical Innovation Foundation, Daegu, Republic of Korea
}

\section{Keywords}

Mouse adenovirus · Recombination-activating gene ·

FVB-Rag2 knockout mice · Systemic infection

\begin{abstract}
Introduction: Recombination-activating gene (Rag) 1 and Rag2, which are essential in V(D)J recombination, play a crucial role in B- and T-cell maturation. Method: We investigated the effects of Rag2 deficiency in clustered regularly interspaced short palindromic repeats/Cas9-mediated FVB-Rag2 knockout (KO) and wild-type (WT) mice infected with mouse adenovirus type 1 (MAV-1) via the intranasal route. Results: MAV-1 infection caused more severe histopathological changes in FVB-Rag2 KO mice than in WT mice. FVB-Rag2 KO mice exhibited moderate to severe inflammation on day 4 and severe inflammation on day 8 post infection. In contrast, WT mice showed mild inflammation on day 4 and mild to severe inflammation on day 8 post infection, including interstitial pneumonia and inflammatory cell infiltration in the lungs and liver. Viral loads in the spleen and kidneys were significantly higher in FVB-Rag2 KO mice than in WT mice on day 8 post infection. Levels of cytokines and chemokines, including macrophage inflammatory protein-1a, induced
\end{abstract}

karger@karger.com www.karger.com/int

Karger!

GOPEN ACCESS
C 2021 The Author(s).

Published by S. Karger AG, Basel

This is an Open Access article licensed under the Creative Commons Attribution-NonCommercial-4.0 International License (CC BY-NC) (http://www.karger.com/Services/OpenAccessLicense), applicable to the online version of the article only. Usage and distribution for commercial purposes requires written permission. protein 10, interferon (IFN)- $\alpha$, IFN- $\gamma$, and tumor necrosis factor alpha, were upregulated in the spleens of FVB-Rag2 KO mice compared with those of WT mice. The upregulation of several cytokines occurred concurrently with the histopathological changes. MAV-1 infection induced more severe systemic infection in FVB-Rag2 KO mice than in WT mice. Conclusion: In mice, Rag2 deficiency induces inflammatory cell recruitment via the upregulation of cytokine and chemokine levels. The MAV-1 infection model can be utilized to assess the efficacy and safety of therapeutic agents for human adenoviral diseases.

(c) 2021 The Author(s)

Published by S. Karger AG, Basel

\section{Introduction}

Mouse adenovirus (MAV) is a nonenveloped, doublestranded DNA virus of the genus Mastadenovirus. Three MAV strains, including MAV type 1 (MAV-1), MAV-2, and MAV-3, can possibly infect laboratory mice $[1,2]$. While experimental infection with MAV-1 did not cause clinical signs in adult immunocompetent mice [3], clinical signs were evident in suckling and immunodeficient mice. In general, MAV-1 induces systemic infection in 
cells of the phagocyte system and microvascular endothelial cells $[4,5]$.

Systemic MAV infection in mice is widely used as a model for studying adenovirus infection in humans $[3,6]$. Systemic MAV infection has been established in outbred [4], inbred [5], and even immunodeficient mice, such as CB-17 severe combined immunodeficiency (SCID) [3] and natural killer (NK) cell-deficient mice [6]. However, overall studies of systemic MAV infection in immunodeficient mice are lacking.

Recombination-activating gene $(R a g) 1$ and Rag2 are essential in $\mathrm{V}(\mathrm{D}) \mathrm{J}$ recombination $[7,8]$, which plays a key role in functional immunoglobulin and $\mathrm{T}$-cell receptor assembly in the early stages of lymphocyte maturation [9]. Rag2 knockout (KO) mice produced using homologous recombination in embryonic stem cells is immunodeficient, with impaired lymphocyte development and lack of immunoglobulin expression [10]. We recently used the clustered regularly interspaced short palindromic repeats/Cas9 system to generated FVB-Rag2 KO mice that lacked mature $\mathrm{T}$ and $\mathrm{B}$ cells and had hardly detectable levels of serum immunoglobulins [11]. In this study, we compared systemic adenovirus infection in FVB-Rag2 $\mathrm{KO}$ and wild-type (WT) mice by intranasally inoculating the mice with MAV-1 to investigate the effects of Rag2 deficiency on systemic MAV-1 infection and to assess whether Rag2 deficiency contributes to the pathogenicity of MAV-1 infection.

\section{Materials and Methods}

\section{Cell and Viral Stock Preparation}

L-929 (NCTC clone 929) mouse fibroblasts were subcultured in Dulbecco's modified Eagle's medium (Gibco, Carlsbad, CA, USA) supplemented with $3.7 \mathrm{~g} / \mathrm{L}$ sodium bicarbonate and $10 \%$ heat-inactivated fetal bovine serum. When the cells reached 70 $80 \%$ confluency $(48 \mathrm{~h}$ ), they were inoculated with MAV-1 strain FL (ATCC stock VR-550). Four days after the inoculation, the culture flask was frozen at $-80^{\circ} \mathrm{C}$ and thawed at room temperature to lyse the cells. Then, the culture flask was centrifuged at 3,000 $\mathrm{g}$ and the supernatant was filtered through a $0.2-\mu \mathrm{m}$ syringe filter. The viral stock was divided into aliquots that were stored at $-80^{\circ} \mathrm{C}$. The virulence of the viral stock was measured using an endpoint dilution assay. The viral titer was determined to be $1.58 \times 10^{4}$ median tissue culture infectious dose/mL.

\section{Animals}

FVB-Rag2 KO mice were created using the clustered regularly interspaced short palindromic repeats/Cas9-mediated gene-targeting system at Yonsei University, Seoul, Republic of Korea [11]. FVBRag2 KO and WT mice were allowed to grow in a laboratory animal breeding room of the College of Veterinary Medicine, Konkuk University, Seoul, Republic of Korea. The mice were housed in individ- ual, ventilated cages with sterilized bedding, food, and water, under controlled conditions of $22 \pm 2^{\circ} \mathrm{C}, 50 \pm 10 \%$ humidity, and a 12 -h light/12-h dark cycle. In the breeding room, pathogen-free conditions are maintained through the use of a barrier system.

\section{$M A V-1$ Inoculation}

Four-week-old FVB-Rag2 KO and WT mice were anesthetized using ketamine $(80 \mathrm{mg} / \mathrm{kg}$ i.p.) and xylazine $(10 \mathrm{mg} / \mathrm{kg}$ i.p. $)$ and intranasally infected with $50 \mu \mathrm{L}$ of MAV-1 $\left(1.74 \times 10^{2}\right.$ tissue culture infectious dose) diluted in sterile phosphate-buffered saline. The body weights of FVB-Rag2 $\mathrm{KO}$ and WT mice were measured daily. The infected mice were sacrificed on day 4 or 8 post infection. The lungs, liver, spleen, and kidneys were harvested for molecular, biological, and histopathological analyses.

\section{Histopathological Examination}

The lung and liver samples were fixed in $10 \%$ neutral buffered formalin and processed to make paraffin-embedded tissue samples that were sliced into $5 \mu \mathrm{m}$-thick sections. The sections were placed on glass slides, deparaffinized, rehydrated, and stained with hematoxylin and eosin. The sections were examined under a BX51 light microscope (Olympus, Tokyo, Japan), and images were captured and analyzed using DP71 software (Olympus).

Histopathological changes in the lungs were scored based on assessment of the following parameters: bronchiolitis ( 0 : absent; 1 : mild; 2: moderate; 3 : severe), alveolar inflammatory cell infiltration (0: absent; 1 : mild; 2 : moderate; 3 : severe), edema (0: absent; 1: present), and endotheliitis ( 0 : absent; 1 : present), as reported [12]. To examine histopathological changes in the liver, a number of inflammatory cell foci were examined in each lobe and the average in 1 lobe was calculated. The histopathological lung and liver scores were determined by 2 researchers.

\section{Immunohistochemical Examination}

Tissue samples were sliced into $5 \mu \mathrm{m}$-thick sections that were placed on silane-coated glass slides. Antigen retrieval was accomplished by boiling the samples in $0.1 \mathrm{M}$ sodium citrate ( $\mathrm{pH}$ 6.0) in a microwave oven. Then, the sections were incubated with $3 \%$ hydrogen peroxide to eliminate endogenous peroxidase activity and with M.O.M. ${ }^{\mathrm{TM}}$ immunoglobulin G blocking reagent (Vector Laboratories, Burlingame, CA, USA) to minimize nonspecific reactions. MAV-1 was detected using MAV mouse-positive sera (CL516 IFA; Charles River Laboratories, Shrewsbury, MA, USA) diluted at a ratio of 1:500, biotinylated anti-mouse immunoglobulin G reagent (Vector Laboratories), and a DAB Peroxidase Substrate Kit (Vector Laboratories). The sections were counterstained with hematoxylin.

\section{Quantification of Viral DNA in Organs}

Equal weights $(0.02 \mathrm{~g})$ of susceptible organs, including the liver, spleen, kidneys, and colon, were harvested, homogenized, and centrifuged $(3,000 \mathrm{~g})$. Viral DNA was isolated using the Viral Gene-spin $^{\mathrm{TM}}$ Viral DNA/RNA Extraction Kit (iNtRON Biotechnology, Seongnam, Republic of Korea). Viral DNA copies were quantified using real-time (q)PCR with standard curve analysis, based on the threshold cycle $(\mathrm{Ct})$ number. qPCRs were run on a CFX96 Real-Time system (Bio-Rad, Hercules, CA, USA) using 1 $\mu \mathrm{L}$ of viral DNA sample from each organ and $\mathbf{i Q}^{\mathrm{TM}} \mathrm{SYBR}^{\circledR}$ Green Supermix (Bio-Rad) according to the manufacturer's protocol. Primers were designed to detect the MAV-1 hexon-coding gene by 
Table 1. Cytokine and chemokine primers sequence

\begin{tabular}{ll}
\hline Target genes & Primer sequence \\
\hline GAPDH & $\begin{array}{l}\text { Forward 5'-AACTTTGGCATTGTGGAAGG-3' } \\
\text { Reverse 5'-ACACATTGGGGGTAGGAACA-3' }\end{array}$ \\
\hline IFN-a & $\begin{array}{l}\text { Forward 5'-CCTGTGTGATGCAACAGGTC-3' } \\
\text { Reverse 5'-TCACTCCTCCTTGCTCAATC-3' }\end{array}$ \\
\hline IFN- - & Forward 5'-TCAAGTGGCATAGATGTGGAAGAA-3' \\
& Reverse 5'-TGGCTCTGCAGGATTTCATG-3' \\
\hline IP-10 & Forward 5'-GAGATCATTGCCACGATGAA-3' \\
\hline MCP-1 & Reverse 5'-CACTGGGTAAAGGGGAGTGA-3' \\
\hline MIP-1a & Revward 5'-CTTCTGGGCCTGCTGTTCA-3' \\
\hline TNF-a & Forward 5'-CCAGCCTACTCATTGGGATCA-3' \\
& Reverse 5'-AGGTCTCTTTGGGATGACACTC-3' \\
\hline
\end{tabular}

IFN, interferon; MIP, macrophage inflammatory protein; MCP, monocyte chemoattractant protein; IP-10, induced protein 10; TNF, tumor necrosis factor alpha; GAPDH, glyceraldehyde 3-phosphate dehydrogenase.

amplifying a fragment of 167 base pairs (bp) in size, as reported [3]. The forward primer was $5^{\prime}$-GGCCAACACTACCGACACTT- $3^{\prime}$ and the reverse primer was $5^{\prime}$-TTTTGTCCTGTGGCATTTGA-3'.

\section{Quantification of Cytokine and Chemokine mRNA Levels in the Spleen}

mRNA levels of the cytokines and chemokines interferon (IFN)- $\alpha$, IFN- $\gamma$, IFN- $\gamma$-induced protein 10 (IP-10), monocyte chemoattractant protein-1 (MCP-1), macrophage inflammatory protein-1 $\alpha$ (MIP-1 $\alpha$ ), and tumor necrosis factor alpha (TNF- $\alpha$ ) in the spleens of infected and uninfected mice were compared using reverse transcription-qPCR. RNA was isolated using TRIzol reagent (Invitrogen, Carlsbad, CA, USA) and $10 \mu \mathrm{L}$ of RNA was reverse-transcribed into $\mathrm{CDNA}$ in a $40-\mu \mathrm{L}$ reaction mixture containing $2 \mu \mathrm{L}$ of oligo-dT $15 \mathrm{bp}$ (50 pmol), $8 \mu \mathrm{L}$ of $2.5 \mathrm{mM} \mathrm{dNTP} \mathrm{(Ta-}$ kara, Otsu, Japan), $8 \mu \mathrm{L}$ of $5 \mathrm{X}$ first-strand buffer (Invitrogen), $4 \mu \mathrm{L}$ of $0.1 \mathrm{M}$ dithiothreitol (Invitrogen), $1 \mu \mathrm{L}$ of RNase OUT (40 unit) (Invitrogen), and $2 \mu \mathrm{L}$ of Moloney murine leukemia virus reverse transcriptase (200 units) (Invitrogen). The cDNA was used as a template for qPCR. The primers used for qPCR are listed in Table 1 . The Ct number for a gene inversely correlates with its expression level. mRNA levels were normalized to the level of glyceraldehyde 3-phosphate dehydrogenase (Gapdh) and were calculated using the $2-\Delta \Delta \mathrm{Ct}$ method.

\section{Statistical Analysis}

Data are expressed as the mean \pm standard deviation. Means of groups were compared using one-way analysis of variance in GraphPad Prism 7.04 (GraphPad Software, San Diego, CA, USA). A $p$ value $<0.05$ was considered significant.

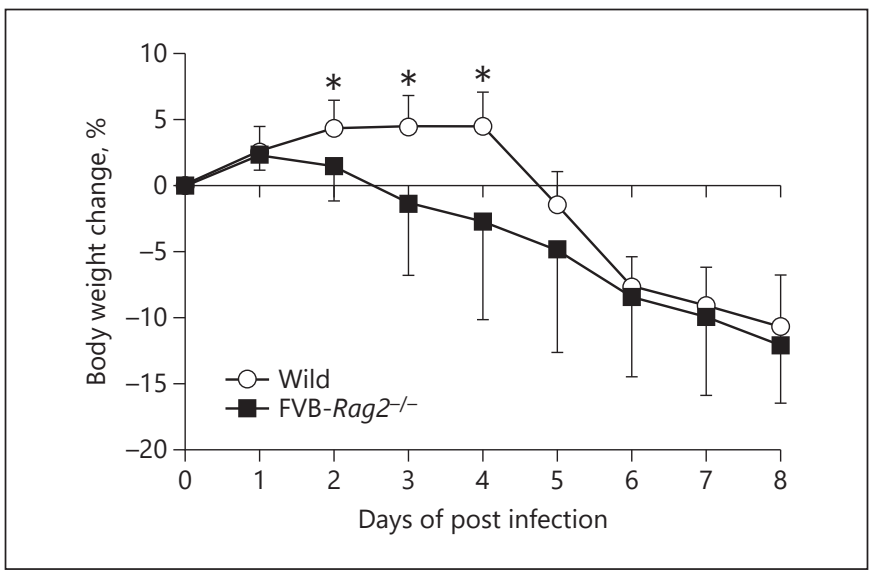

Fig. 1. Body weight changes in FVB-Rag2 KO (FVB-Rag $\left.{ }^{-/}\right)$and WT mice. All mice in both groups $(n=6)$ were inoculated intranasally with $1.74 \times 10^{2}$ TCID $_{50}$ of MAV-1. Body weights of FVBRag2 KO and WT mice were measured daily. ${ }^{*} p<0.05$ versus WT mice at the indicated time points. KO, knockout; WT, wild-type; $\mathrm{TCID}_{50}$, tissue culture infectious dose; MAV-1, mouse adenovirus type 1; Rag, recombination-activating gene.

\section{Results}

\section{Body Weight Changes}

After MAV-1 infection, the body weights of WT mice increased until day 4 and then gradually decreased, whereas FVB-Rag2 KO mice gradually decreased in weight after day 1 . Compared to that in WT mice, the rate of body weight loss (\%) between days 2 and 4 post infection was significantly higher in FVB-Rag2 $\mathrm{KO}$ mice (Fig. 1). None of the mice died before day 8 post infection.

\section{Viral Loads in Tissues of Rag2 KO and WT Mice \\ Infected with $M A V-1$}

To determine the difference in susceptibility of FVBRag2 KO and WT mice to MAV-1 infection, MAV-1 DNA copy numbers in different organs of the mice were measured using qPCR. Viral DNA copy numbers in the spleen of FVB-Rag2 $\mathrm{KO}$ mice on day 8 post infection were significantly higher $(\sim 23$-fold $)$ than those in WT mice (Fig. 2a). As expected, the highest viral loads were noted on day 8 post infection. In the liver, viral DNA copy numbers were significantly higher in both FVB-Rag2 KO and WT mice on day 8 post infection ( $\sim 5$-fold) than that in WT mice on day 4 post infection (b). Viral DNA copy numbers in the kidneys of FVBRag2 KO mice were significantly higher ( 3-fold) than those in WT mice on day 8 post infection (Fig. 2c). In the colon of FVB-Rag2 KO mice, viral DNA copy num- 


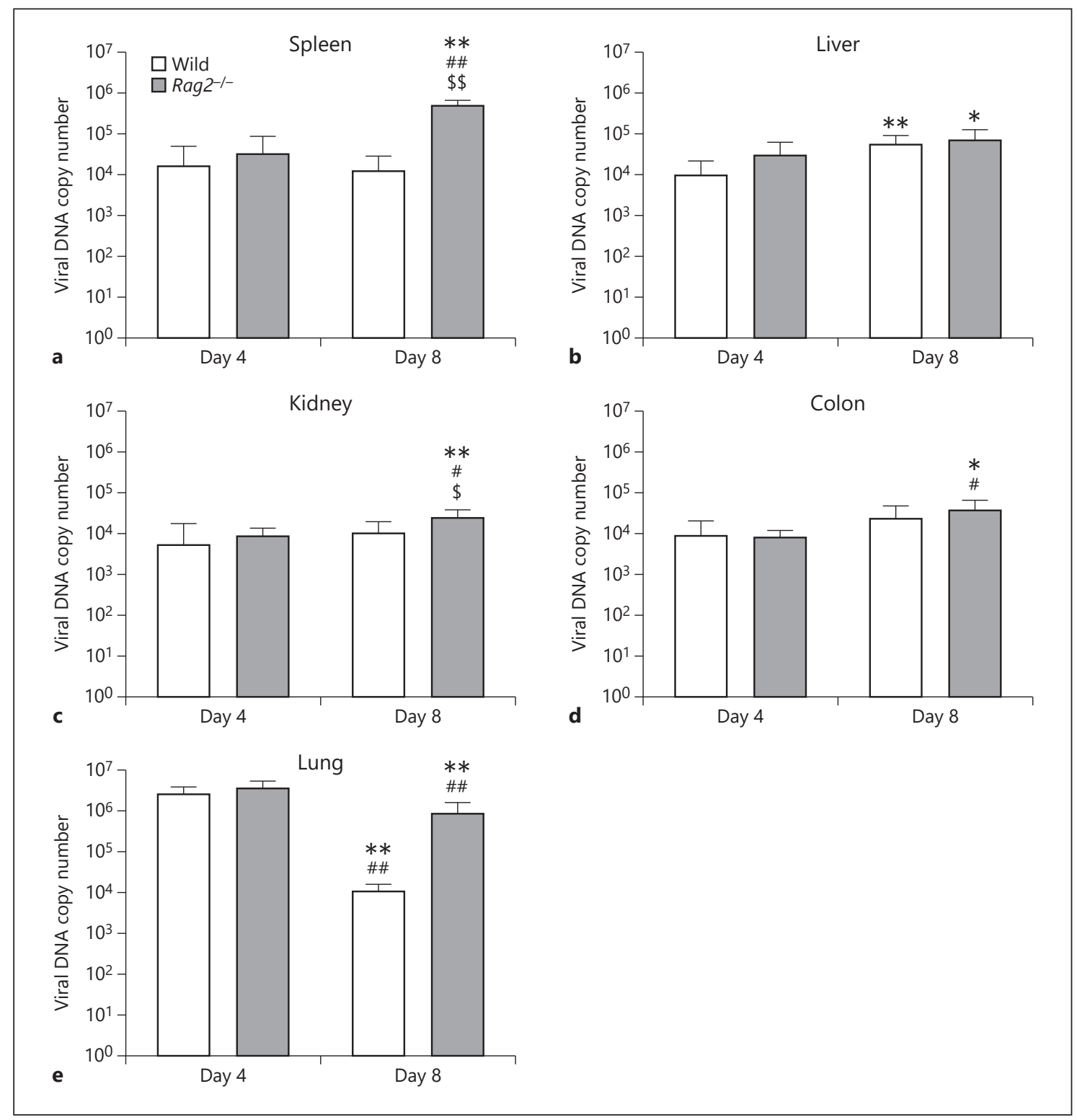

Fig. 2. Viral DNA copy numbers in FVB-Rag2 KO (FVB-Rag2 $2^{-/-}$) and WT mice. All mice in both groups $(n=6)$ were inoculated intranasally with $1.74 \times 10^{2} \mathrm{TCID}_{50}$ of MAV-1. Viral DNA copy numbers per $\mathrm{mg}$ of tissue were measured in the spleen (a), liver (b), kidneys (c), colon (d), and lungs (e) on days 4 and 8 post infection. Data are presented as the mean \pm SD. ${ }^{*} p<0.05,{ }^{* *} p<0.01$

Fig. 3. Representative images of hematoxylin and eosin-stained sections (a-f) and histopathological scores $(\mathbf{g}, \mathbf{h})$. Inflammatory cell infiltrates in the perivascular and peribronchial space (arrows) and alveolar septa (asterisks) of the lungs were detected in both WT (a, c) and FVB-Rag2 KO (FVB-Rag $\left.2^{-/-}\right)(\mathbf{b}, \mathbf{d})$ mice on day 4 $(\mathbf{a}, \mathbf{b})$ and $8(\mathbf{c}, \mathbf{d})$ post infection. Inflammatory cell foci (arrow heads) in the liver were detected in WT (e) and FVB-Rag2 KO (f) versus WT mice on day 4 post infection; ${ }^{*} p<0.05$ and ${ }^{\# \#} p<0.01$ versus $\operatorname{Rag}^{-/-}$on day 4 post infection; ${ }^{\$} p<0.05$ and ${ }^{\$ \$} p<0.01$ versus WT mice on day 8 post infection. KO, knockout; WT, wildtype; TCID $_{50}$, tissue culture infectious dose; MAV-1, mouse adenovirus type 1; Rag, recombination-activating gene; SD, standard deviation. mice on day 8 post infection. Inflammation scores for the lungs ( $\mathbf{g})$ and inflammatory foci in the liver (h). Data are presented as the mean \pm SD. ${ }^{*} p<0.05,{ }^{* *} p<0.01$ versus WT mice on day 4 post infection; ${ }^{\#} p<0.05$ and ${ }^{\# \#} p<0.01$ versus $\operatorname{Rag}^{-/}{ }^{--}$mice on day 4 post infection. KO, knockout; WT, wild-type; Rag, recombination-activating gene; SD, standard deviation.

(For figure see next page.) 


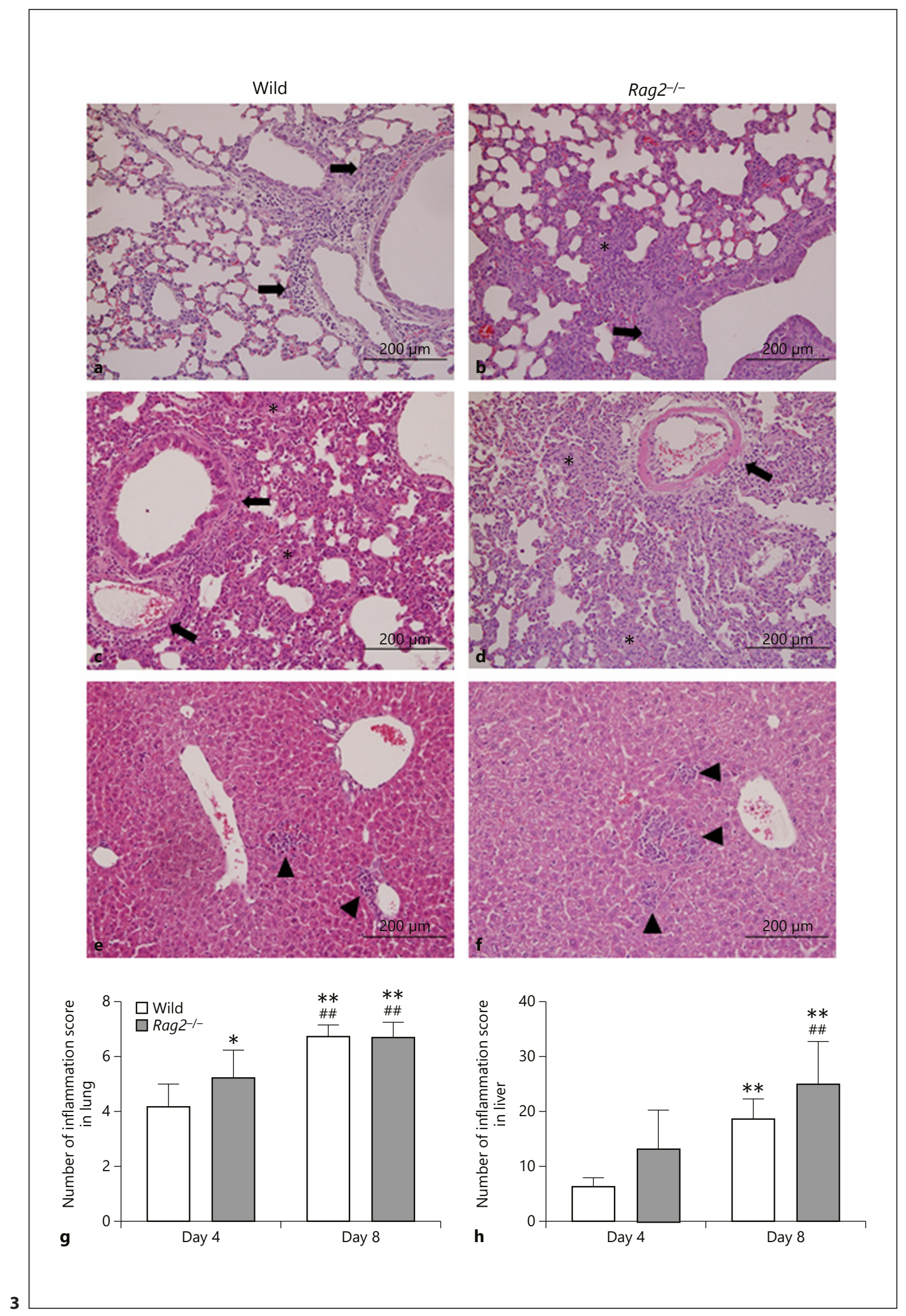


Fig. 4. Representative images of immunohistochemical staining. MAV-1 viral antigens (arrows) were detected in the lungs (a, b), liver (c, d), and splenic white pulp $(\mathbf{e}, \mathbf{f})$ in both WT (a, c, e) and FVB-Rag2 $\mathrm{KO}$ $\left(\mathrm{FVB}-\mathrm{Rag}^{-/-}\right)(\mathbf{b}, \mathbf{d}, \mathbf{f})$ mice on day 8 post infection. KO, knockout; WT, wild-type; MAV-1, mouse adenovirus type 1; Rag, recombination-activating gene.

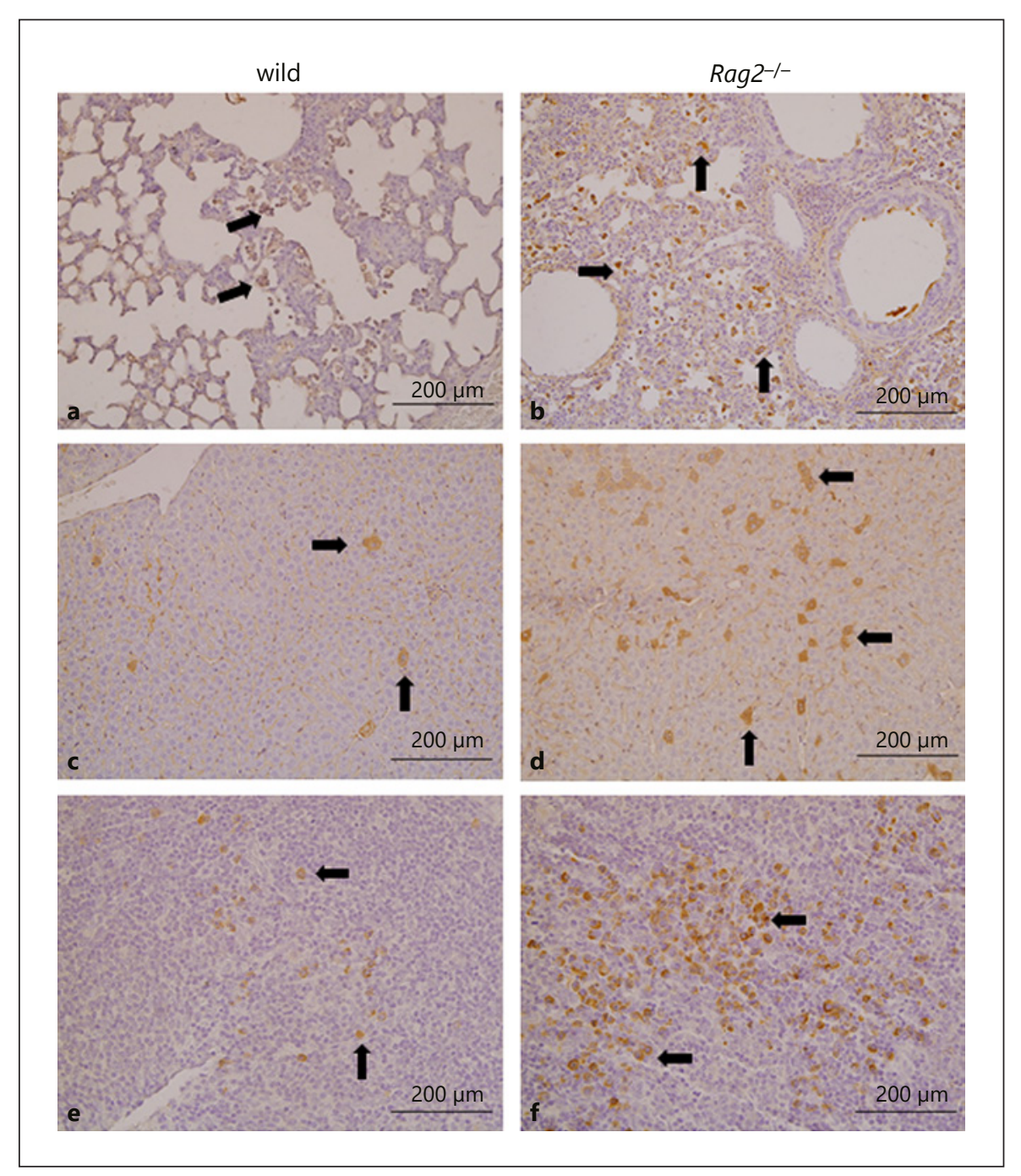

bers were significantly increased ( $\sim 4$-fold) on day 8 post infection compared to those in both FVB-Rag2 KO and WT mice on day 4 post infection (Fig. 2d). On day 8 post infection, WT mice had lower $(\sim 80$-fold $)$ viral loads in the lungs than FVB-Rag2 KO mice. Viral loads in the lungs of WT mice on day 4 post infection were significantly lower ( $\sim 250$-fold) than those on day 8 post infection (Fig. 2e). Among all organs evaluated, the lungs were found to be the most susceptible to MAV-1 infection. In FVB-Rag2 KO mice, MAV-1 infection susceptibility of the various organs increased in the following order: lungs, spleen, liver, colon, and kidneys.

\section{Histopathological and Immunohistochemical Analyses}

Mononuclear cell infiltration in the lungs was observed in the perivascular and peribronchial spaces (Fig. 3a-d) in both FVB-Rag2 KO and WT mice on days
4 and 8 post infection. Alveolar walls were thickened by the infiltrating mononuclear cells in FVB-Rag2 $\mathrm{KO}$ mice on days 4 and 8 post infection (Fig. 3b, d). In WT mice, interstitial pneumonia was observed only on day 8 post infection (Fig. 3c). Edema and vascular endotheliitis were occasionally observed in the lungs of FVB-Rag2 $\mathrm{KO}$ and WT mice (data not shown). Lung inflammation scores of FVB-Rag2 KO mice on days 4 and 8 post infection were significantly higher than those of WT mice on day 4 post infection. In addition, lung inflammation scores of FVBRag2 KO and WT mice were significantly higher on day 8 than on day 4 post infection (Fig. 3g).

In the liver, a few foci composed of infiltrating mononuclear cells were seen in both FVB-Rag2 $\mathrm{KO}$ and WT mice on days 4 and 8 after infection (Fig. 3e, f). FVB-Rag2 $\mathrm{KO}$ mice had significantly more inflammatory foci in the liver on day 8 post infection than WT mice on day 4 post 

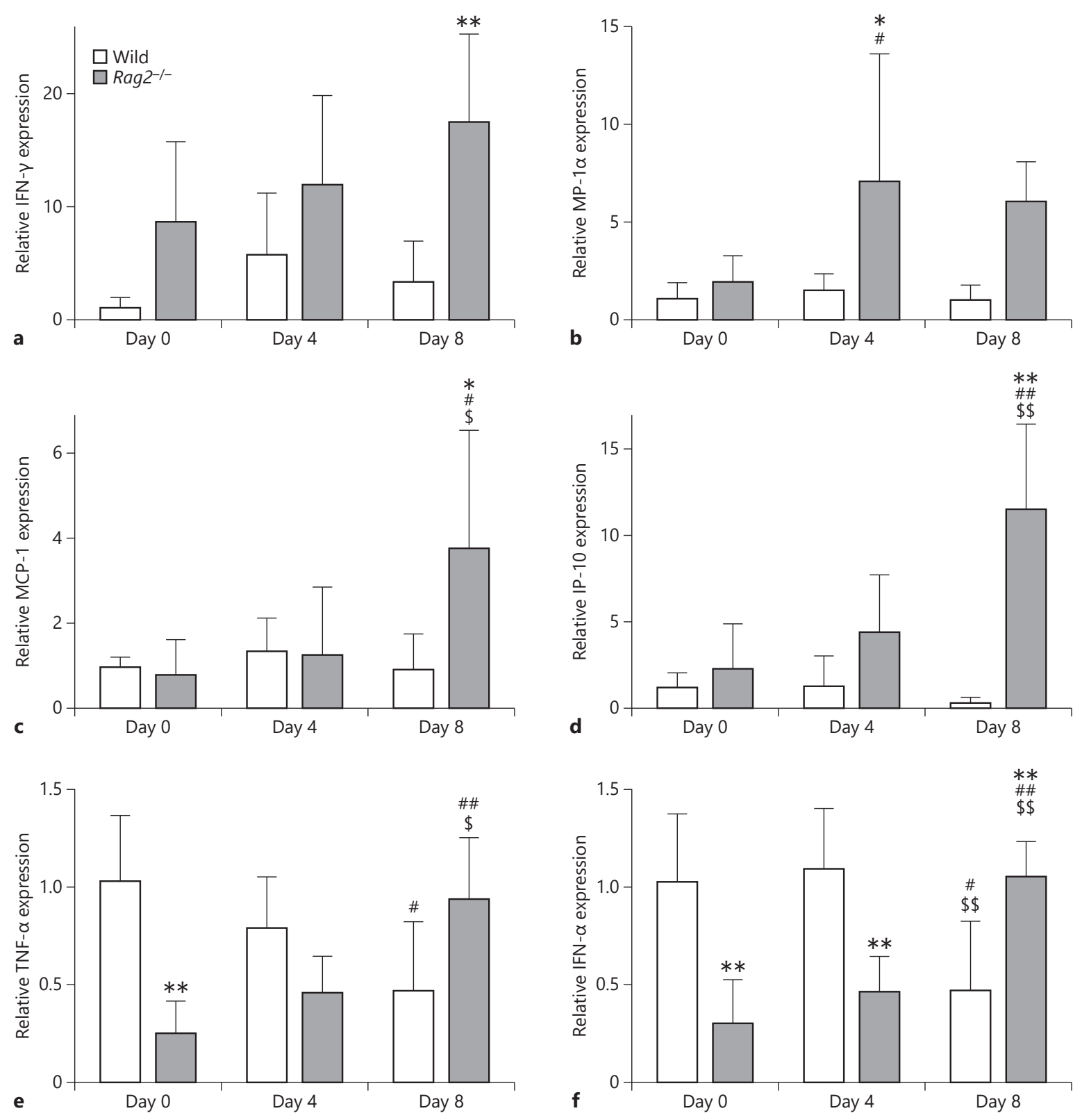

Fig. 5. Cytokine mRNA levels in the spleens of FVB-Rag2 $\mathrm{KO}$ and WT mice. IFN- $\gamma$ (a), MIP-1 $\alpha$ (b), MCP-1 (c), IP-10 (d), TNF- $\alpha$ (e), and IFN- $\alpha(\mathbf{f})$ mRNA levels $(n=6)$ were measured on days 4 and 8 post infection using RT-qPCR. Expression levels were normalized to Gapdh expression. Data are presented as the mean \pm SD. ${ }^{*} p$ $<0.05,{ }^{* *} p<0.01$ versus WT at the indicated time points; ${ }^{*} p<0.05$, ${ }^{\# \#} p<0.01$ versus day 0 for the same genotype; ${ }^{\$} p<0.05,{ }^{\$ \$} p<0.01$

versus day 4 for the same genotype. KO, knockout; WT, wild-type; Rag, recombination-activating gene; IFN, interferon; MIP, macrophage inflammatory protein; MCP, monocyte chemoattractant protein; IP-10, induced protein 10; TNF, tumor necrosis factor alpha; RT, reverse transcription; Gapdh, glyceraldehyde 3-phosphate dehydrogenase; SD, standard deviation.

infection. In addition, the numbers of inflammatory foci in the liver were significantly higher in FVB-Rag2 $\mathrm{KO}$ and WT mice on day 8 than on day 4 post infection (Fig. $3 \mathrm{~h}$ ).

Immunohistochemical analysis was used to detect the presence of MAV-1 antigens in the organ tissues. In the lungs, MAV-1 antigens were observed in inflammatory 
cells infiltrated in the alveolar space and septa in both FVB-Rag2 KO and WT mice on day 8 post infection (Fig. 4a, b). Hepatocytes in the liver and mononuclear cells in the splenic white pulp of FVB-Rag2 KO and WT mice were positive for MAV-1 antigens on day 8 post infection (Fig. 4c-f). Interestingly, many MAV-1 antigens were detected in the lungs (Fig. 4b), liver (Fig. 4d), and splenic white pulp of FVB-Rag2 $\mathrm{KO}$ mice when compared to WT mice (Fig. 4f). In addition, MAV-1-positive cells were observed in the lamina propria of the small intestine and colon and in the mesenteric lymph nodes (data not shown).

Inflammatory Cytokine Levels Are Increased in MAV1-Infected Rag2 KO Mice

To understand the difference in susceptibility of FVBRag2 KO and WT mice to MAV-1 infection, we examined changes in cytokine expression levels after MAV-1 infection in spleen homogenates. At baseline (day 0), FVBRag2 KO showed higher expression of IFN- $\gamma$, but lower expression of IFN- $\alpha$ and TNF- $\alpha$ than WT mice. In FVBRag2 KO mice, the mRNA levels of cytokines were significantly increased on day 8 post infection compared to day 0 (MCP-1, $p=0.017$; IP-10, $p<0.0001$; IFN- $\alpha, p<$ 0.009; Fig. 5c, d, f). In contrast, in WT mice, all cytokines tested were either decreased in expression or exhibited no change on day 8 post infection compared to day 0 (TNF- $\alpha$, $p=0.019$ : IFN- $\alpha, p=0.013$; Fig. 5 e, f). On day 8 post infection with MAV-1, the mRNA levels of IFN- $\gamma$, MCP-1, IP-10, and IFN- $\alpha$ were significantly increased in FVBRag2 KO mice compared to WT mice (Fig. 5a, c, d, f).

\section{Discussion}

Life-threatening systemic MAV-1 infection has been reported in a SCID mouse model with a BALB/c background; the mice died within 17-19 days post infection due to focal hemorrhagic enteritis [3]. In the present study, we investigated the effect of Rag2 deficiency on MAV-1 infection by using FVB-Rag2 KO mice. We assessed MAV-1 infection in the immunodeficient mice on days 4 and 8 post infection. Throughout the experimental period, no deaths were noted in WT and FVB-Rag2 KO mice.

Body weight changes indicate the body condition of infected animals. In this study, MAV-1 infection caused a decrease in body weight between days 5 and 8 post infection both in FVB-Rag2 $\mathrm{KO}$ and WT mice. The rate of body weight loss was significantly higher in FVB-Rag2

Rag2 Deficiency Enhances Susceptibility to MAV-1
KO mice than in WT mice between days 3 and 4 post infection. These results suggest that Rag2 deficiency exacerbates infection-related body weight loss in the early stage (day 4). CD8 T cells play a crucial role in the early stage of viral infections, and several studies have suggested that CD8 T-cell deficiency is related to body weight loss induced by respiratory viral infections $[13,14]$. However, in 1 study, CD8 deficiency was found to provide protection against body weight loss induced by MAV-1 infection [15]. Thus, the association between body weight loss and immune deficiency, including CD8 cell deficiency, or Rag2 deficiency in MAV-1 infection remains controversial.

Further, we observed histopathological changes in the lungs and liver to compare the susceptibility of FVB-Rag2 $\mathrm{KO}$ and WT mice to MAV-1 infection. In the lungs, we observed typical histopathological findings of viral pneumonia, such as inflammation, peribronchiolar edema, and thickened alveolar walls [16]. In the early stage, MAV-1 infection induced mild inflammation in the lungs and liver. In the later stage (day 8), the mild infection progressed to severe infection both in FVB-Rag $2 \mathrm{KO}$ and WT mice. The histopathological changes induced by MAV-1 infection in the early stage were more severe in FVB-Rag2 KO mice than in WT mice. These differences in the early stage of infection were in accordance with the delayed body weight change in WT mice.

To further assess the protective effect of Rag2 in systemic MAV-1 infection, we compared the viral load, as a marker of susceptibility, in various organs of FVB-Rag2 $\mathrm{KO}$ and WT mice. Viral DNA copy numbers were increased in all organs except the lungs on day 8 compared to day 4 post infection both in FVB-Rag2 $\mathrm{KO}$ and WT mice. FVB-Rag2 $\mathrm{KO}$ mice had higher viral loads in the spleen and kidneys than WT mice on day 8 post infection. WT mice showed viral clearance in the lungs on day 8 post infection, although viral pneumonia was increased. However, FVB-Rag2 KO mice showed no viral clearance on day 8 post infection. These results indicate that FVBRag2 KO mice are more susceptible to MAV-1 infection than WT mice. The detection of viral DNA in the various organs indicates that after intranasal infection, viremia occurs and the virus disseminates into the target organs. This observation is in line with findings in a study of MAV systemic infection in outbred mice, in which the presence of the virus in the lungs, kidneys, spleen, liver, and other organs was demonstrated using in situ hybridization [4]. B cells reportedly protect against disseminated MAV-1 infection [17]. The role of B lymphocytes in MAV-1 infection may explain the significant difference 
in viral DNA loads between WT and FVB-Rag2 KO mice in this study.

The expression levels of cytokines and chemokines, including IFN- $\gamma$, MIP-1 $\alpha$, MCP-1, IP-10, and IFN- $\alpha$, were upregulated in the spleens of FVB-Rag2 KO mice during the late stage of infection with MAV-1. However, in WT mice, the levels of these chemokines remained unchanged or were decreased on day 8 post infection. Similarly, chemokine upregulation has been reported in the lungs of mice intranasally infected with murine gamma herpes virus 68 [18] and influenza virus [19]. In MAV-1-infected SCID mice, TNF- $\alpha$, IL- $1 \beta$, MIP- $1 \alpha$, and MIP- $1 \beta$ levels were upregulated in infected tissues, including the intestine and liver [3]. Immunohistochemical analysis revealed the presence of MAV-1 antigens in mononuclear cells that had infiltrated into the lungs, liver, and splenic white pulp. The numbers of MAV-1-positive cells were higher in FVB-Rag2 KO mice than in WT mice. These mononuclear cells are the major sources of cytokines and chemokines. MIP-1a primarily induces the migration of macrophages, monocytes, neutrophils, and NK cells [20, 21]. Monocytes and macrophages are the major sources of MCP-1 secretion for antiviral responses in the peripheral circulation $[22,23]$. MCP-1 is a chemoattractant of NK cells $[24,25]$. IP-10 is a chemoattractant of macrophages, dendritic cells, NK cells, and activated T lymphocytes [26], which are related to viral infections and NKcell differentiation. Thus, the enhanced upregulation of cytokines and chemokines in FVB-Rag2 $\mathrm{KO}$ mice may induce enhanced systematic inflammatory cell recruitment in these mice.

\section{Conclusion}

A MAV-1 systemic infection model was established in FVB-Rag2 KO mice. This MAV-1 systemic infection model can be utilized in future to assess the efficacy and safety of therapeutic agents in human adenoviral diseases. Further, we found that Rag2 deficiency may induce inflammatory cell recruitment via the upregulation of cytokines and chemokines.

\section{Statement of Ethics}

All animal studies were approved by the Institutional Animal Care and Use Committee of Konkuk University (KU18099).

\section{Conflict of Interest Statement}

The authors have no conflicts of interest to declare.

\section{Funding Sources}

This work was supported by Korea Institute of Planning and Evaluation for Technology in Food, Agriculture, Forestry (IPET) through the Agriculture, Food, and Rural Affairs Convergence Technologies Program for Educating Creative Global Leader Program, funded by the Ministry of Agriculture, Food and Rural Affairs (MAFRA) (320005041SB080).

\section{Author Contributions}

H.-K. Lee, Y.-K. Choi, and E.-S. Jeong conceived and designed the study. H.-K. Lee and Y.-K. Choi analyzed the data and wrote the manuscript. H.-K. Lee, S.-M. Seo, J.-Y. Kim, and H.-W. Kim performed the experiments. All the authors read and approved the manuscript.

\section{Data Availability Statement}

All data generated or analyzed during this study are included in this article. Further inquiries can be directed to the corresponding author.

\section{References}

1 Baker DG. Natural pathogens of laboratory mice, rats, and rabbits and their effects on research. Clin Microbiol Rev. 1998 Apr;11(2): 231-66.

2 Hemmi S, Spindler KR. Murine adenoviruses: tools for studying adenovirus pathogenesis in a natural host. FEBS Lett. 2019 Dec;593(24): 3649-59.

3 Lenaerts L, Verbeken E, De Clercq E, Naesens L. Mouse adenovirus type 1 infection in SCID mice: an experimental model for antiviral therapy of systemic adenovirus infections. Antimicrob Agents Chemother. 2005 Nov; 49(11):4689-99.

4 Kajon AE, Brown CC, Spindler KR. Distribution of mouse adenovirus type 1 in intraperitoneally and intranasally infected adult outbred mice. J Virol. 1998 Feb;72(2):1219-23.

5 Charles PC, Guida JD, Brosnan CF, Horwitz MS. Mouse adenovirus type- 1 replication is restricted to vascular endothelium in the CNS of susceptible strains of mice. Virology. 1998 Jun;245(2):216-28.

6 Welton AR, Gralinski LE, Spindler KR Mouse adenovirus type 1 infection of natural killer cell-deficient mice. Virology. 2008 Mar; 373(1):163-70.

7 Schatz DG, Oettinger MA, Baltimore D. The $\mathrm{V}(\mathrm{D}) \mathrm{J}$ recombination activating gene, RAG1. Cell. 1989 Dec;59(6):1035-48. 
8 Oettinger MA, Schatz DG, Gorka C, Baltimore D. RAG-1 and RAG-2, adjacent genes that synergistically activate $\mathrm{V}(\mathrm{D}) \mathrm{J}$ recombination. Science. 1990 Jun;248(4962):1517-23.

9 Han S, Zheng B, Schatz DG, Spanopoulou E, Kelsoe G. Neoteny in lymphocytes: Rag1 and Rag2 expression in germinal center B cells. Science. 1996 Dec;274(5295):2094-7.

10 Shinkai Y, Rathbun G, Lam KP, Oltz EM, Stewart V, Mendelsohn M, et al. RAG-2-deficient mice lack mature lymphocytes owing to inability to initiate $\mathrm{V}(\mathrm{D}) \mathrm{J}$ rearrangement. Cell. 1992 Mar;68(5):855-67.

11 Lee JH, Park JH, Nam TW, Seo SM, Kim JY, Lee HK, et al. Differences between immunodeficient mice generated by classical gene targeting and CRISPR/Cas9-mediated gene knockout. Transgenic Res. 2018 Jun;27(3): $241-51$.

12 Jeon HY, Park JH, Park JI, Kim JY, Seo SM, Ham SH, et al. Cooperative interactions between toll-like receptor 2 and toll-like receptor 4 in murine Klebsiella pneumoniae infections. J Microbiol Biotechnol. 2017 Aug; 27(8):1529-38.

13 Frey S, Krempl CD, Schmitt-Gräff A, Ehl S. Role of T cells in virus control and disease after infection with pneumonia virus of mice. J Virol. 2008 Dec;82(23):11619-27.
14 Hassert M, Harris MG, Brien JD, Pinto AK. Identification of protective CD8 $\mathrm{T}$ cell responses in a mouse model of Zika virus infection. Front Immunol. 2019 Jul;10:1678.

15 Molloy CT, Andonian JS, Seltzer HM, Procario MC, Watson ME Jr, Weinberg JB. Contributions of CD8 $\mathrm{T}$ cells to the pathogenesis of mouse adenovirus type 1 respiratory infection. Virology. 2017 Jul;507:64-74.

16 Pritt BS, Aubry MC. Histopathology of viral infections of the lung. Semin Diagn Pathol. 2017 Nov;34(6):510-7.

17 Moore ML, McKissic EL, Brown CC, Wilkinson JE, Spindler KR. Fatal disseminated mouse adenovirus type 1 infection in mice lacking B cells or Bruton's tyrosine kinase. J Virol. 2004 Jun;78(11):5584-90.

18 Weinberg JB, Lutzke ML, Efstathiou S, Kunkel SL, Rochford R. Elevated chemokine responses are maintained in lungs after clearance of viral infection. J Virol. 2002 Oct; 76(20):10518-23.

19 Wareing MD, Lyon AB, Lu B, Gerard C, Sarawar SR. Chemokine expression during the development and resolution of a pulmonary leukocyte response to influenza A virus infection in mice. J Leukoc Biol. 2004 Oct;76(4): 886-95.

20 Cook DN. The role of MIP-1 alpha in inflammation and hematopoiesis. J Leukoc Biol. 1996 Jan;59(1):61-6.
21 Guan E, Wang J, Norcross MA. Identification of human macrophage inflammatory proteins 1alpha and 1 beta as a native secreted heterodimer. J Biol Chem. 2001 Apr;276(15): 12404-9.

22 Yoshimura T, Robinson EA, Tanaka S, Appella E, Kuratsu J, Leonard EJ. Purification and amino acid analysis of two human glioma-derived monocyte chemoattractants. J Exp Med. 1989 Apr;169(4):1449-59.

23 Yoshimura T, Yuhki N, Moore SK, Appella E, Lerman MI, Leonard EJ. Human monocyte chemoattractant protein-1 (MCP-1). Fulllength cDNA cloning, expression in mitogenstimulated blood mononuclear leukocytes, and sequence similarity to mouse competence gene JE. FEBS Lett. 1989 Feb;244(2):487-93.

24 Deshmane SL, Kremlev S, Amini S, Sawaya BE. Monocyte chemoattractant protein-1 (MCP-1): an overview. J Interferon Cytokine Res. 2009 Jun;29(6):313-26.

25 Mahad DJ, Ransohoff RM. The role of MCP-1 (CCL2) and CCR2 in multiple sclerosis and experimental autoimmune encephalomyelitis (EAE). Semin Immunol. 2003 Feb;15(1):2332.

26 Romagnani S. The Th1/Th2 paradigm. Immunol Today. $1997 \mathrm{Feb}$;15(1):263-6. 